\title{
Editorial
}

\section{Bioinspired Functional Materials}

\author{
Yongmei Zheng, ${ }^{1}$ Jingxia Wang, ${ }^{2}$ Yongping Hou, ${ }^{1}$ Hao Bai, ${ }^{3}$ and Michael Z. $\mathrm{Hu}^{4}$ \\ ${ }^{1}$ Key Laboratory of Bio-Inspired Smart Interfacial Science and Technology of Ministry of Education, \\ School of Chemistry and Environment, Beihang University, Xueyuan Road 37, Haidian District, Beijing 100191, China \\ ${ }^{2}$ Key Laboratory of Green Printing, Institute of Chemistry, Chinese Academy of Sciences, Beijing 100190, China \\ ${ }^{3}$ Lawrence Berkeley National Laboratory, 1 Cyclotron Road, MS 62-0227, Berkeley, CA 94720, USA \\ ${ }^{4}$ Oak Ridge National Laboratory, Oak Ridge, TN 37831, USA \\ Correspondence should be addressed to Yongmei Zheng; zhengym@buaa.edu.cn
}

Received 20 October 2014; Accepted 20 October 2014; Published 25 November 2014

Copyright (C) 2014 Yongmei Zheng et al. This is an open access article distributed under the Creative Commons Attribution License, which permits unrestricted use, distribution, and reproduction in any medium, provided the original work is properly cited.

This special issue is focused on the nanoscale or micro/nanoscale structures similar to the biological features in multilevels or hierarchy and so on. Research by mimicking biological systems has shown more impact on many applications due to the well-designed micro-/nanostructures inspired from the biological surfaces or interfaces; thus, the materials may achieve the fascinating functionality. The bioinspired functional materials may be fabricated by developing novel technology or methods such as synthesis, selfassembly, and soft lithography at micro- or nanolevel or multilevels and, in addition, the multidisciplinary procedures of physical or chemical methods and nanotechnology to mimic the biological multiscale micro-/nanostructures onto one-/two-dimensional surface materials.

This issue collected these researched works from biological to bioinspired materials as follows.

J. Meng et al. revealed the microstructure of a novel fibrous protein: solenin from Solen grandis ligament and identified the protein by MALDI-TOF-TOF-MS and LCMS-MS analyses and found that the protein fiber has no hierarchical structure and is homologous to keratin type II cytoskeletal 1 and type I cytoskeletal 9-like, containing "SGGG," "SYGSGGG," "GS," and "GSS" repeat sequences; the $\beta$-sheet structure and those repeat sequences which form "glycine loops" may give solenin excellence elastic and flexible properties to withstand tensile stress caused by repeating opening and closing of the shell valves in vivo. This paper contributes a novel fibrous protein for the protein materials world.

S. Kashyap et al. reported the in situ visualization of a novel acidic bacterial recombinant protein, MamC, commonly present in the magnetosome membrane of several magnetotactic bacteria, including Magnetococcus marinus, strain MC-1. The findings provided an insight into the selfassembly of MamC and point to formation of the extended protein surface, which is assumed to play an important role in the formation of biotemplated inorganic nanoparticles. The self-organization of MamC is compared to the behavior of another acidic recombinant iron-binding protein, Mms6.

Z. Cao et al. reviewed the article on scaffolding biomaterials for cartilage regeneration. The use of tissue engineering approach in which scaffolds play a vital role to regenerate cartilage has become a new research field. Investigating the advances in biological cartilage scaffolds has been regarded as the main research direction and has great significance for the construction of artificial cartilage. Native biological materials and synthetic polymeric materials have their advantages and disadvantages. The disadvantages can be overcome through either physical modification or biochemical modification. Additionally, developing composite materials, biomimetic materials, and nanomaterials can make scaffolds acquire better biocompatibility and mechanical adaptability.

$\mathrm{S}$. Hou et al. reported a new alumina membrane with hour-glass shaped nanochannels by using the double-side anodization method and the subsequently in situ pore opening procedure, which is applied to develop the tunable ionic current rectification devices that were modulated by ions gradient. By regulating the $\mathrm{pH}$ gradient, tunable ionic current rectification properties which are mainly dependent on the asymmetric surface charge density or polarity distribution on the inner walls of the nanochannels can be obtained. Such specific alumina nanochannels would be considered as 
a promising candidate for building bioinspired artificial ion channel systems.

M. I. Hassan et al. reported the bioactivity assessment of poly ( $\varepsilon$-caprolactone)/hydroxyapatite electrospun fibers for bone tissue engineering application. The fiber diameter is entangled by the concentration of PCL with some adjustment of parameters during electrospinning process. PCL with lower concentration had bead structure while higher concentration had smooth fiber. The fiber diameter of PCL was increased with the addition of nHA. The immersion of PCL/nHA fiber in simulated body fluid (SBF) had bonelike apatite layer on its surface while PCL showed no results. PCL/nHA showed high water uptake and had improved wettability compared to PCL alone, suggesting that PCL/nHA fibers were more hydrophilic than PCL fiber.

T. Zhao et al. presented the preparation of phase-change polymer colloidal photonic crystals (PCs) by assembling hollow latex spheres encapsulated with dodecanol for the first time. The monodispersed hollow latex spheres were obtained by phase reversion of monodispersed core-shell latex spheres in the n-hexane, which dissolves the PS core and retains the PMMA/PAA shell. The as-prepared phase-change colloidal PCs show stable phase-change behavior. This fabrication of phase-change colloidal PCs would be significant for PC's applications in functional coatings and various optic devices.

M. Park et al. reported a method of creating a biomimetic functional biointerface which was introduced to enhance human neural stem cell (hNSC) adhesion. The hNSCcompatible biointerface was prepared by the oxidative polymerization of the neurotransmitter norepinephrine, which generates a nanoscale organic thin layer, termed poly (norepinephrine) (pNE). The pNE-coated biointerfaces provided a highly favorable microenvironment for hNSCs, with no observed cytotoxicity. The adhesive properties of pNE interfaces led to micropatterns of cellular attachment, thereby demonstrating the ability of the interface to organize the stem cells. This highly facile surface-modification method using a biomimetic pNE thin layer can be applied to a number of suitable materials that were previously not compatible with hNSC technology.

N. Wang et al. reported variable responsive wettability films via electrospinning induced by solvents. The three kinds of interesting distinct wettability films are fabricated by a facile electrospinning technique. The films are composites of poly(N-isopropylacrylamide) and polystyrene but different precursor solvents. By taking advantage of the conformational changes of poly( $\mathrm{N}$-isopropylacrylamide) chains and polystyrene chains in different solvents, the films exhibit responsive wettability variation to temperature.

M. Gonzalez et al. reported the effect of silver nanofibers on the deformation properties of blood vessels: towards the development of new nanotechnologies to prevent rupture of aneurysms. They proposed an innovative approach to prevent the rupture of an aneurysm based on the use of nanotechnology to improve the strength of the blood vessel. They presented results on the effect of silver nanofibers on the resistance toward deformation of blood vessels. The silver nanofibers are grown on the surface of the blood vessels. Treatment of blood vessels with silver nanofibers is a potential translational clinical tool for preventing rupture of aneurysms in a clinical setting.

W. Zhao et al. prepared the preparation of aminomodified PAN fibers with triethylenetetramine as aminating reagents and their application in $\mathrm{CO}_{2}$ adsorption. The aminomodified polyacrylonitrile (AMPAN) fiber was synthesized by the reaction of polyacrylonitrile (PAN) fiber and triethylenetetramine (TETA) in the presence of water. The effect of water amount, reaction temperature, and time on the weight increase degree and alkali content of the AMPAN fiber was investigated in detail. The characterization results indicated that TETA could be grafted onto the surface of PAN fiber by this method.

\section{Acknowledgments}

The editors thank the authors for their efforts and time spent for each manuscript. The lead editor thanks all editors for the time spent in reviewing, assigning reviews, and commenting on submitted manuscripts. The editors hope that this special issue will be useful to investigators in interfacial functional materials. 

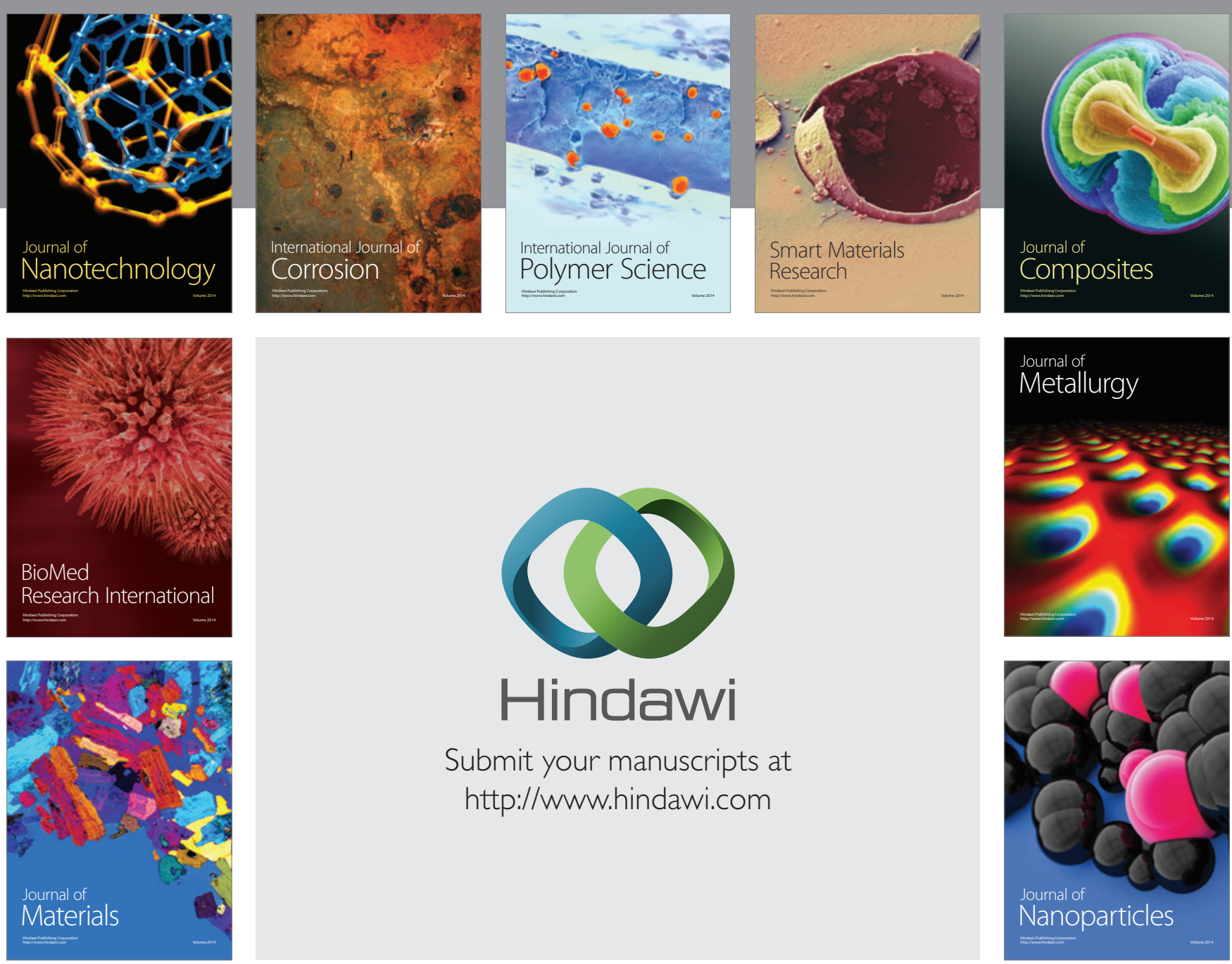

Submit your manuscripts at http://www.hindawi.com
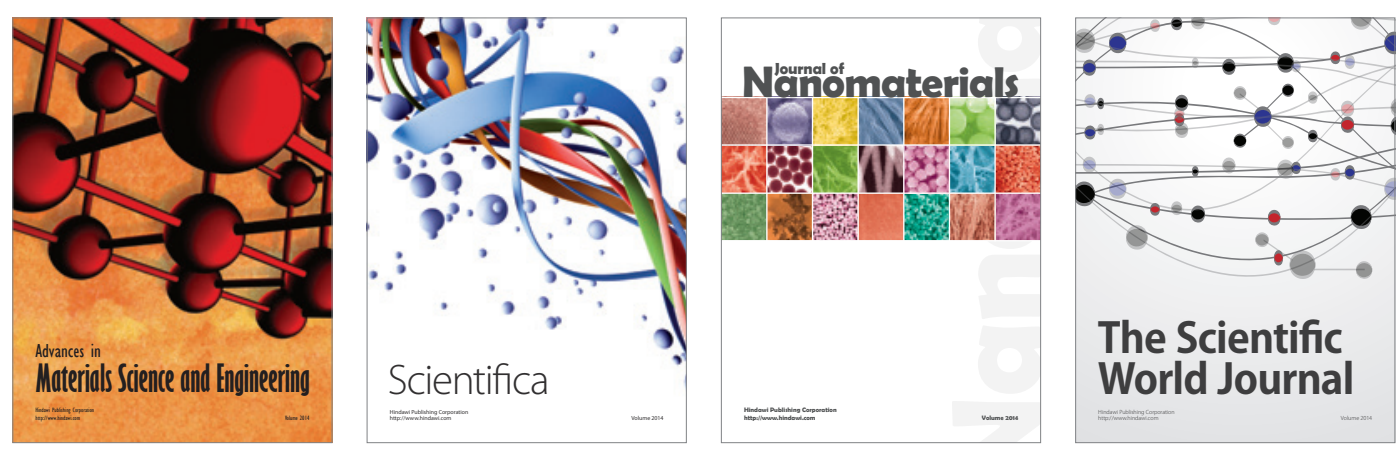

\section{The Scientific World Journal}
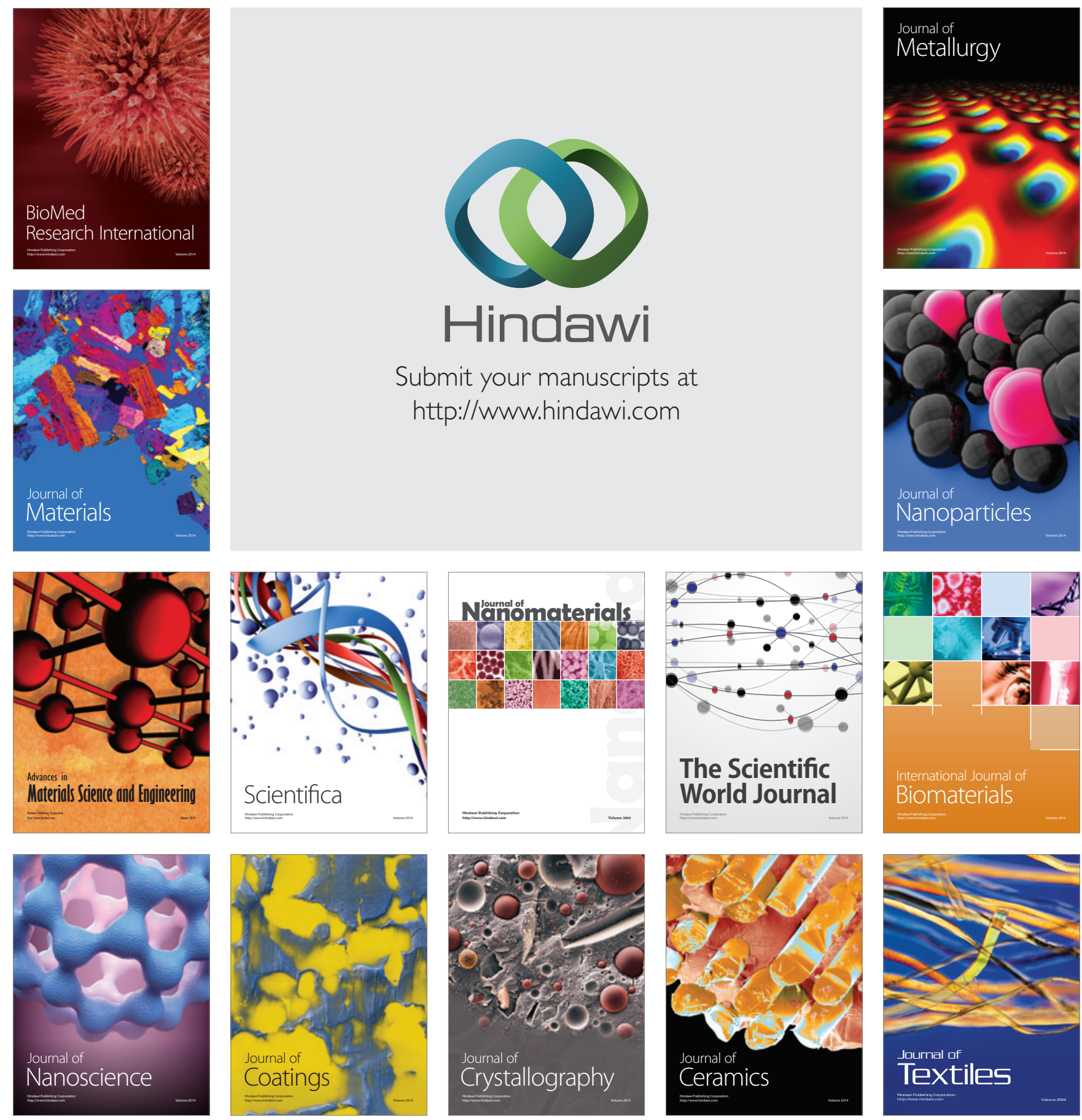